\title{
Balancing and blade-shaping of a single-blade impeller
}

\author{
Matej Kurilla ${ }^{1, *}$, Branislav Knížat ${ }^{1}$, Róbert Olšiak ${ }^{1}$, Pavol Slovák ${ }^{1}$, and Maroš Hyriak ${ }^{2}$ \\ ${ }^{1}$ Slovak University of Technology in Bratislava, Nám. slobody 17, 81231 Bratislava, Slovakia \\ 2 PRAKTIKPUMP, Jesenského 63, 96001 Zvolen, Slovakia
}

Received: 27 March 2019 / Accepted: 5 October 2019

\begin{abstract}
Single-blade centrifugal pumps with a screw-shaped blade are widely used to pump slurry water containing solids and fibrous matters. These hydraulic machines should be frequently used, because of their suitable hydraulic and mechanical properties, in many industrial areas. There cannot be applied a conventional method to design a single-blade impeller, due to its lack of symmetry. The designing of an impeller, which enables fulfilled main requests such as good hydraulic, mechanical properties and the ball passage diameter as well as the influence of the blade outlet angle on hydraulic parameters is described in this academic paper.
\end{abstract}

Keywords: Single-blade impeller / balancing / pump / characteristic curves

\section{Introduction}

Screw centrifugal pumps are specifically designed to pump slurry water containing solids and fibrous matters. They represent an important segment of hydraulic machines in the wastewater pumping area. Many kinds of viscous, dualphase, non-homogeneous and solid-liquid media can be pumped. Single-blade centrifugal pump characteristic features are high efficiency, steep characteristic, low NPSH and a flat power curve [1]. A large solid passage diameter enables fragile materials such as fish, fruit, and vegetables to be pumped safely. Due to unique blade geometry, the screw pump can be characterized as a non-clog, no winding and damage-resistant device. The whole pump construction is generally simple and massive. The impeller is made of nodular iron with high abrasive resistance, which minimizes life-cycle costs and ensures a long lifetime and high reliability [2]. This paper addresses the impact of a blade outlet angle change on hydraulic parameters and the hydraulic parameter comparison of a balanced and unbalanced impeller of such a pump using CFD simulations and laboratory measurements. It widens considerably poor knowledge about single-blade impeller. The results presented in this paper show the marked difference in flow through the single-blade channel to flow through the multiblade channel, but it is an only small step to understand the complex unsteady flow through screw-shaped blade impeller.

\footnotetext{
* e-mail: matej.kurilla@stuba.sk
}

\section{Flow in a single-blade impeller}

Flow through a single-blade impeller has several specific features that differentiate it from flow through a conventional centrifugal pump with multi-blade impeller. A singleblade impeller is characterized by a long wrap angle (about $500^{\circ}$ ) and a wide flow channel. The conventional multi-blade impeller (usual with 7 blades) enables better to regulate the direction of the flow thereby the orientation of the flow velocity vector is more similar to the blade surface tangent. Due to the wide channel and only single blade water in the single-blade channel flows through without continuous contact with the blade surface and the offset between the flow velocity vector and the blade surface tangent is more significant than that of the multi-blade impeller. It might results in back-flows, the boundary layer breakage, which occurs, to a lesser extent, also in a multi-blade impeller, and subsequently in the hydraulic losses increasing.

The single-blade impeller geometry should be carefully proposed with respect to a smooth flow through it, which means no sudden changes in the flow direction. Hydraulic losses are therefore kept to a minimum possible level and achievable efficiency might rise to a multi-blade pump level.

Because of a long wrap angle, the blade outlet angle accomplishes relatively low values and as a result, the Q-Y curves are considerably steep and also linear, resulting in the stability during the pump operation. Due to the single blade, the impeller is geometrically asymmetric with respect to the rotational axis. The same applies to a diffuser, which is the volute casing. 
Rotational, asymmetrical pressure and velocity fields interact with an also non-symmetrical fluxional field in the stator. Accordingly, non-zero radial thrust acts on an impeller, even in the design point. The radial thrust increases along with a higher blade outlet angle and also with higher flow rates [3]. The radial force problem is thereby stronger than that of conventional centrifugal pumps. Considerable researcher's effort is focused on the knowledge of energy transformation mechanism in singleblade pumps. The aim is decreasing of losses and therefore growth of efficiency. According to the fluxional field asymmetry, the substantial performance parameter fluctuation in the pump during one revolution can be observed. Performance parameters are torque, specific energy, flow rate, and shaft power.

Because of standard design methods usage [4] the designing process does not provide an accurate methodology as necessary. Consequently these methods are combined with CFD computation methods [5]. We consider a shape of the dimensionless function of specific energy, in the design process - equation (1):

$$
\Psi=\Psi_{t}-\Theta\left(\Phi_{2}\right)
$$

The dimensionless flow rate $\Phi_{2}$ and theoretical specific energy $\Psi_{\mathrm{t}}$ are given by the following equations [6]:

$$
\begin{gathered}
\Phi_{2}=\frac{Q}{\eta_{V} A_{2} U_{2}}, \\
\Psi_{t}=\sigma_{Y}-\frac{\Phi_{2}}{\tan \beta_{2}} .
\end{gathered}
$$

In equations (1)-(3) $\Psi_{\mathrm{t}}-$ theoretical specific energy, $\beta_{2}$ - blade outlet angle, $\eta_{\mathrm{V}}-$ volume efficiency, $A_{2}$ - outlet area, $U_{2}$ - tangential outlet velocity.

The curve $\Psi-\Phi_{2}$, respectively $Y-Q$ (specific energyflow rate) is shaped by the $\sigma_{\mathrm{Y}}$ and $\theta\left(\Phi_{2}\right)$ parameter, i.e., the slip factor [6] and the hydraulic losses function.

The slip factor of the flow in the impeller $\sigma_{Y}$, in equation (3), is about 0.4 for single-blade impellers. It characterizes imperfect guidance of a liquid [7]. We can estimate hydraulic losses in the optimal point based on the supposed efficiency of the pump.

A subject of the proposal is meridional cut of the pump as well as a shape of the blade. The considerably important fact has to be considered here, in contrast with conventional centrifugal pumps. Owing to the asymmetric blade shape the computed blade designed to fulfil energetic parameters causes dynamic unbalance of the whole rotational part of the pump. The impeller loses its mechanical properties as a rotor. Accordingly, the impeller has to be balanced by adding additional material. It influences retroactively the shape of the blade channel and the whole hydraulic solution. We will refer to the influence of the blade outlet angle change and the impeller dynamic balancing on hydraulic parameters of the pump in this paper.
Table 1. Single-blade pump hydraulic parameters.

\begin{tabular}{lll}
\hline Parameter & Value & Description \\
\hline$Q\left[\mathrm{~m}^{3} / \mathrm{s}\right]$ & 0.011 & Flow rate \\
$Y[\mathrm{~J} / \mathrm{kg}]$ & 105 & Specific energy \\
$n\left[\mathrm{~min}^{-1}\right]$ & 2900 & Speed \\
$n_{\mathrm{b}}[-]$ & 0.15 & Specific speed \\
\hline
\end{tabular}

\subsection{Hydraulic parameters of the pump}

A single-blade pump, with hydraulic parameters shown in Table 1, was used for testing the hydraulic properties of researched single-blade impellers. Four impeller alternatives, marked as $\mathrm{B}_{1}, \mathrm{~B}_{2}$ and $\mathrm{B}_{3}, \mathrm{~B}_{4}$, were created in order to fulfil given parameters (Tab. 1). All impellers have the same meridional cut so the main dimensions, particularly $\mathrm{D}_{1}$ and $\mathrm{D}_{2}$ dimensions, equal by all. The impellers $\mathrm{B}_{1}$ and $\mathrm{B}_{2}$ with different blade mass distribution and different blade outlet angle allow the blade outlet angle course influence on the hydraulic parameters to be researched. The impellers $\mathrm{B}_{3}$ and $\mathrm{B}_{4}$ are hydraulically identical and the added material influence on the hydraulic properties can be observed. Although the $\mathrm{B}_{3}$ and $\mathrm{B}_{4}$ blades consist of the same planes on the suction sides, the pressure sides are different. The $\mathrm{B}_{3}$ impeller blade is designed as the expandable line surface with the constant thickness of $5 \mathrm{~mm}$ and the $\mathrm{B}_{4}$ impeller blade shape is additionally changed so the mechanical request of the dynamic balancing is fulfilled. Different designing approaches lead to creation of the unbalance impeller $\mathrm{B}_{3}$ and the dynamically balanced impeller $\mathrm{B}_{4}$. Owing to the ball passage diameter request, which is $50 \mathrm{~mm}$, the suction and the discharge diameter of the pump is $50 \mathrm{~mm}$ and all internal spaces take into account the request. This allows the ball with the diameter of $50 \mathrm{~mm}$ pass through the pump without any damage. The expected hydraulic efficiency range of such kind of the single-blade pump lies between 50 and $60 \%$. Clean water was used as the pumped medium during the laboratory measurements and the CFD simulations.

Welded metal plates were used for manufacturing the spiral casing.

\subsection{Geometric parameters of impellers}

The whole pump was engineered according to the requested parameters mentioned in Table 1 . Two impeller alternatives, hereinafter referred to as impeller $\mathrm{B}_{1}$ and impeller $\mathrm{B}_{2}$ were proposed to fulfil given parameters. Both hydraulic alternatives were CFD tested in order to find out their hydraulic properties during the research at this level. Till the hydraulic final shape investigation of impellers is not brought to an end, mechanical properties are not solved so impeller $\mathrm{B}_{1}$, as well as impeller $\mathrm{B}_{2}$, is not dynamically balanced so far. All designing (geometric) parameters are shown in Table 2.

The impellers $\left(\mathrm{B}_{1}\right.$ and $\left.\mathrm{B}_{2}\right)$ are shown in Figure 1 . There is marked difference not only in blade outlet angle but also 

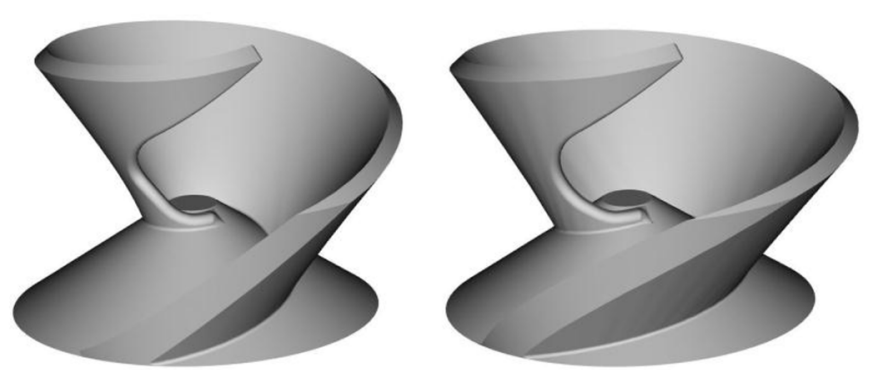

Fig. 1. Impeller $B_{1}$ (left) and impeller $B_{2}$ (right).
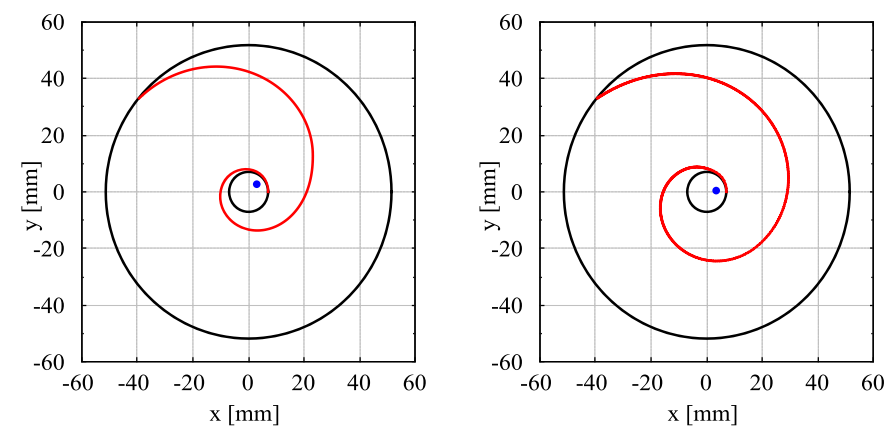

Fig. 2. Blade mass placement on the back shroud - impeller $B_{1}$ (left) and impeller $\mathrm{B}_{2}$ (right).

Table 2. Impeller $\mathrm{B}_{1}$ and $\mathrm{B}_{2}$-geometric parameters.

\begin{tabular}{|c|c|c|c|}
\hline & $\begin{array}{l}\text { Impeller } \\
\mathrm{B}_{1}\end{array}$ & $\begin{array}{l}\text { Impeller } \\
\mathrm{B}_{2}\end{array}$ & Description \\
\hline$D_{1}[\mathrm{~mm}]$ & \multicolumn{2}{|c|}{50} & Inlet diameter \\
\hline$D_{2}[\mathrm{~mm}]$ & \multicolumn{2}{|c|}{138} & Outlet diameter \\
\hline$\beta_{2}\left[^{\circ}\right]$ & 8 & 20 & Blade outlet angle \\
\hline$\varphi_{2}\left[^{\circ}\right]$ & \multicolumn{2}{|c|}{500} & Wrap angle \\
\hline Balanced & \multicolumn{2}{|c|}{ No } & Dynamic balancing \\
\hline
\end{tabular}

in the blade mass placement on the back shroud, which affects the relative blade angle course.

A view, parallel with the rotational axis, on the back shroud, is in Figure 2. The red solid line represents the blade mass and the blue dot responds to the centre of gravity location.

A difference between the impeller $\mathrm{B}_{1}$ geometry and the impeller $\mathrm{B}_{2}$ geometry is obvious, as well as the centre of gravity movement depending on the blade mass distribution. The CFD analysis will be carried out to find out the influence on the hydraulic properties of such pumps.

The influence of balancing process on the mechanical and hydraulic properties can be seen on the impeller $\mathrm{B}_{3}$ and the impeller $\mathrm{B}_{4}$. The suction sides of the impeller blades are identical expandable line surfaces and also the meridional cut dimensions are the same. The hydraulically identical impellers are designed as semi-opened.

However, the impeller $\mathrm{B}_{3}$ mechanical request of a balancing is not fulfilled, thus the impeller $\mathrm{B}_{3}$ is statically

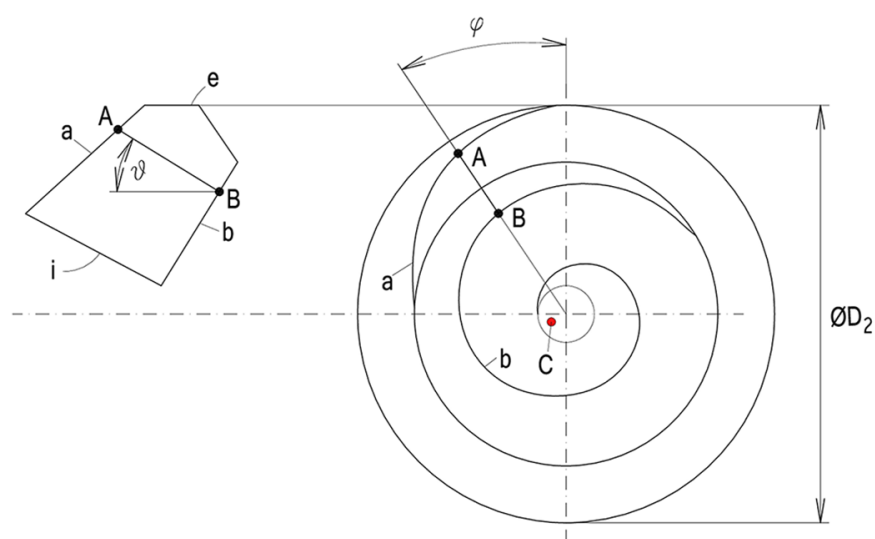

Fig. 3. Basic blade shape as the expandable line surface.

Table 3. Impeller $\mathrm{B}_{3}$ and $\mathrm{B}_{4}$-geometric parameters.

\begin{tabular}{|c|c|c|c|}
\hline & $\begin{array}{l}\text { Impeller } \\
\mathrm{B}_{3}\end{array}$ & $\begin{array}{l}\text { Impeller } \\
\mathrm{B}_{4}\end{array}$ & Description \\
\hline$\overline{D_{1}[\mathrm{~mm}]}$ & \multicolumn{2}{|c|}{50} & Inlet diameter \\
\hline$D_{2}[\mathrm{~mm}]$ & \multicolumn{2}{|c|}{138} & Outlet diameter \\
\hline$\beta_{2}\left[^{\circ}\right]$ & \multicolumn{2}{|c|}{8.2} & Outlet blade angle \\
\hline$\varphi_{2}\left[{ }^{\circ}\right]$ & \multicolumn{2}{|c|}{486} & Wrap angle \\
\hline Balanced & No & Yes & Dynamic balancing \\
\hline
\end{tabular}

and dynamically unbalanced, the hydraulic parameters are considerably good because the blade channel is shaped directly according to the calculation. Figure 3 illustrates the basic blade shape of the impeller $\mathrm{B}_{3}$ as the expandable line surface. The centre of gravity location of the impeller $\mathrm{B}_{3}$ is marked as the red point $\mathrm{C}$. The variable inclination of cut lines A-B, represented by the angle $\vartheta$, enables the impeller mass distribution to be changed in order to get the centre of gravity as close as possible to the rotational axis of the pump. Although this designing approach allows the change of the centre of gravity location during the impeller designing, the blade shaping cannot totally balance the impeller and the additional material has to be added to dynamically balance the impeller. Described designing method is explained in Figure 3 where the curves a and $b$ are streamlines on the front side of the impeller meridional cut (a) and on the hub of the impeller (b).

Table 3 shows the geometric parameters of the impellers $\mathrm{B}_{3}$ and $\mathrm{B}_{4}$. The pictures of both impellers are in Figure 4. Two different technologies are applied to manufacture the impellers. Whereas the impeller $B_{3}$ is made of welded steel plates, the impeller $\mathrm{B}_{4}$ is printed on a $3 \mathrm{D}$ printer directly from a $\mathrm{CAD}$ file and the used material is the ABS plastic.

Both impellers are designed so the solid passage diameter is up to $50 \mathrm{~mm}$. It means the pump is nonclogging for the solids with the diameter up to $50 \mathrm{~mm}$. The impeller $\mathrm{B}_{4}$ meets totally balance requests so the rotational axis passes through the impeller centre of gravity (static 

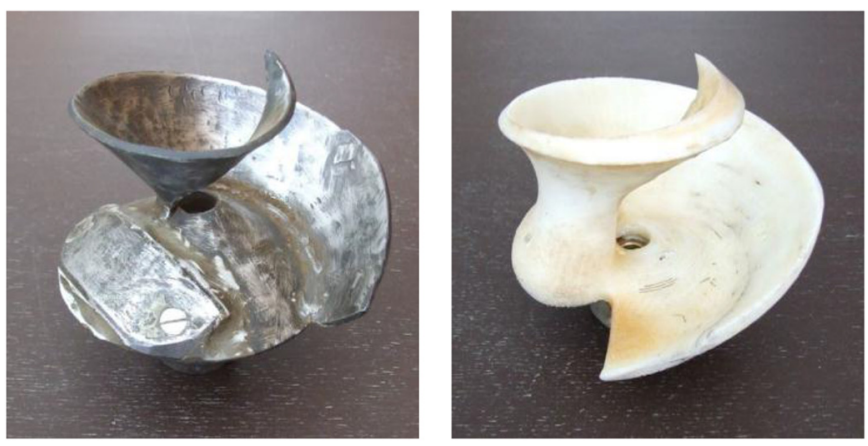

Fig. 4. Impeller $B_{3}$ (left) and impeller $B_{4}$ (right).
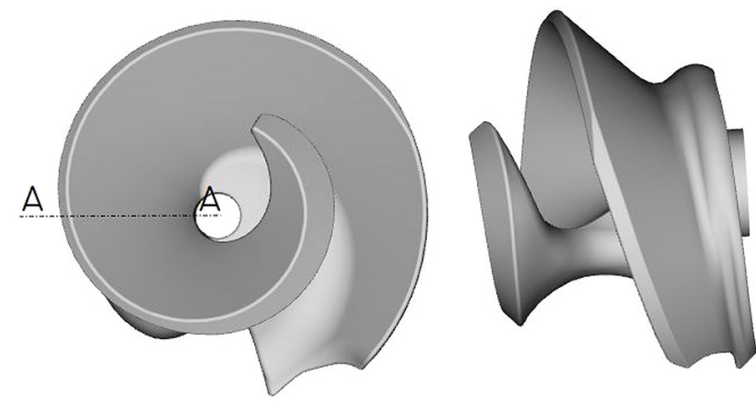

Fig. 5. Totally balanced impeller $\mathrm{B}_{4}-$ result of balancing process.

balance) and the main impeller inertia axis lies on the rotational axis (dynamic balance).

CAD software is used for additional balancing. This process consists of adding or removing material from the 3D impeller model. Due to the remaining hydraulic parameters and the ball passage diameter of the pump the balancing mass is added carefully in areas with no impact on hydraulic conditions of flow. The additional material can be seen in Figure 5. The cut A-A indicates the area with most significant geometry changes as it is obvious also from Figure 4.

\subsection{Laboratory measurement test facility}

A physical model was used for testing the hydraulic parameters of proposed hydraulic solutions of the impellers. A certain modification of the standard measurement method of hydrodynamic pump performance characteristics according to STN EN ISO 9906 was applied [8]. The experimental station scheme, including the measure and control system connections, is in Figure 6. Owing to the low specific energy provided by the single-blade pump the hydraulic resistance in the test rig should be reduced to the minimal possible level in order to enable the performance characteristic measurement in the area of higher flow rates than the $Q_{\mathrm{opt}}$ one. The connection of the suction side of the tested pump (TP) to the water-filled reservoir (R1) decreased the hydraulic resistant in the test rig. The constant water level $\mathrm{z} 1$ in the $\mathrm{R} 1$ reservoir was ensured by the additional pumping circuit (AP) and also by the weir (W).

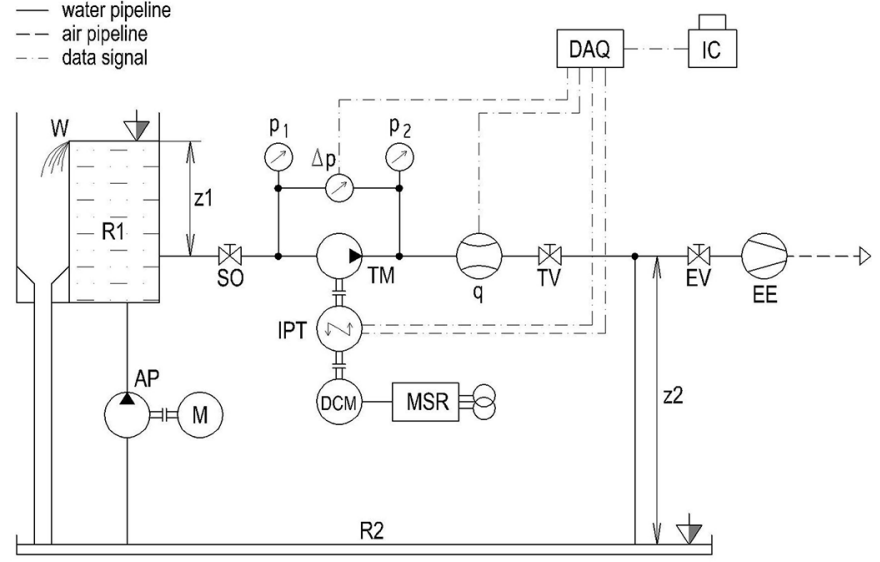

Fig. 6. Scheme of the experimental station.

The tested pump pressure side was connected by a pipeline to the reservoir 2 (R2). Due to the low water level in the $\mathrm{R} 2$ reservoir, which was lower than the rotational axis of the tested model about the height of $\mathrm{z} 2$, the evacuation circuit (EE) was part of the test rig. The EE circuit, which consisted of the water ring vacuum pump and the evacuation valve (EV), served as a vacuum source and filled the test rig pipelines with the pumped medium (water). The evacuation valve function was to close the pipeline after the water ring vacuum pump has filled the pipelines with water and the throttling valve (TV) was used for controlling the operational parameters. In order to attain low pressure drop in the test rig the transit time ultrasonic flow meter (q), which has no loss of pressure when dry transducers are used, was applied. The direct current motor (DCM) with the thyristor motor speed regulator (MSR) was connected to the three-phase power supply and powered the tested model.

AP-additive pump, DAQ-data acquisition, DCMdirect current motor, EE-evacuation equipment (water ring vacuum pump), EV-evacuation valve (ball), ICindustrial measuring computer, IPT-input power transducer, MSR-motor speed regulator, $p 1$-inlet pressure gauge, $p 2$-outlet pressure gauge, $\Delta p$-differential pressure transducer, $q$-flowmeter, R1-reservoir 1, R2-reservoir 2, SO-shut off valve, TM-tested model, TV-throttling valve, W-weir.

The results of model pump measurements are $Y=f(Q)$ and $\eta=f(Q)$ curves, graphically presented in Figure 8. The construction of such performance characteristics requires the specification of a measured variable vector and the subsequent calculation. The measured variable vector can be defined as follows: $p 1$, pressure at the inlet of the tested pump; $p 2$, pressure at the outlet of the tested pump; $\Delta p$, pressure difference (inlet-outlet); $Q$, flow rate; $\mathrm{Mk}$, torque at the pump shaft; $n$, rotational speed.

A wiring diagram of the sensor and transducer system, which was used for measuring the defined variables, can be seen in Figure 6. Analog signals were brought to the analogue-to-digital converter (DAQ) and the DAQ converter was connected to the measuring and operational computer (IC) with the USB data bus. The DasyLAB 
Table 4. Technical specification of measurement technology.

\begin{tabular}{lllll}
\hline Parameter & Sensor/transducer type & Measure range & Measuring signal full range & Accuracy full scale \\
\hline Pressure differential $\Delta p$ & Capacitance & $0-139 \mathrm{kPa}$ & $4-20 \mathrm{~mA}$ & $0.25 \%$ \\
Flow rate & Ultrasonic transit time & $0-15 \mathrm{l} / \mathrm{s}$ & $4-20 \mathrm{~mA}$ & $1 \%$ \\
Torque & Strain gauge & $0-20 \mathrm{Nm}$ & $0-10 \mathrm{VDC}$ & $0.5 \%$ \\
Rotational speed & Inductive & $0-3000 \mathrm{rev} / \mathrm{min}$ & $0-10 \mathrm{VDC}$ & $0.25 \%$ \\
A/D Converter & Sigma delta & $0-20 \mathrm{~mA} /$ & $24 \mathrm{bit}$ resolution & $0.05 \% \pm 1 \mathrm{LSB}$ \\
& & $0-10 \mathrm{~V}$ & & \\
\hline
\end{tabular}

software provided collection of digitalised data and measurement management. Table 4 shows the basic technical specification of the used measurement technology.

A point by point measurement method of the performance characteristics was used: every single $j$-point of the characteristic responded to the $j$-parameter value according to the adjusted $\mathrm{Q} j$ value (flow rate). Changes of the throttling valve hydraulic resistant let to changes of the Q $j$ flow rates provided by the tested pump. Measurements were carried out with the constant rotational speed of the pump shaft: $n=$ const. Because of random error occurrence, the measurement of every $j$-point was repeated $n$-times, to reduce the random error influence, and the resulting value of measured variable was set as the arithmetic mean:

$$
\bar{x}=\frac{\sum_{i=1}^{n} x_{i}}{n} .
$$

Possible rotational speed deviation, from the required one, affected values of the measured physical variables. In order to achieve correct results possible hydraulic parameter deviations caused by rotational speed deviations were recalculated on the basis of the affinity laws.

$$
\begin{gathered}
Y_{j}=\Delta \bar{p}_{j} \bar{Q}_{j} \\
P_{j}=\bar{M}_{k j} 2 \pi \bar{n}_{j} \\
\eta_{j}=\frac{Y_{j}}{P_{j}} .
\end{gathered}
$$

\section{Results and discussion}

The numerical analysis using CFD methods was carried out to find out the hydraulic properties of impellers $B_{1}$ and $B_{2}$. On the one hand, hydraulic parameters of impellers $\mathrm{B}_{3}$ and $\mathrm{B}_{4}$ were researched numerically using CFD methods and, on the other hand, experimentally.

The CFD simulation model of the pump was divided into three domains: suction pipe, impeller, spiral casing. The commercial software Ansys - CFX V15 was used for generating the mesh, creating the computational model and for carrying out the numerical computations. The mesh was generated as an unstructured in the Ansys Meshing package and consisted of tetrahedral elements. Sizing criteria were applied in order to attain balance between result accuracy and computing time. Owing to the covering of all CFD pump model internal surfaces with a continuous inflation layer the proximity advanced size function was applied. The CFD impeller models of $\mathrm{B}_{1}, \mathrm{~B}_{2}$ and $\mathrm{B}_{3}$ pump were similar in element number. The whole pump model consisted of approximately 7 million elements (impeller - 3.1 million; spiral casing -2.2 million; suction pipe -1.7 million). Because of added and removed material the $\mathrm{B}_{4} \mathrm{CFD}$ model was more complicated so a substantial increase of element number has been observed. The $\mathrm{B}_{4}$ CFD model consisted of 19.2 million elements (impeller 15.2 million; spiral casing - 2.1 million; suction pipe 1.9 million).

The numerical simulations were carried out as steady state using the SST turbulence model with the Frozen Rotor frame change option. A mass flow rate was set as the boundary condition at the inlet to change the operation point of the impeller and the average static pressure of $1 \mathrm{~atm}$ was defined as the boundary condition at the outlet. Usage of the steady-state CFD model with the Frozen Rotor interfaces caused a stator/rotor clocking effect, which could decrease the result accuracy. To eliminate this negative effect the impellers were sequentially turned in three various positions. The results of simulations were averaged. The CFD analysis was carried out only in the close mass flow rate range to the optimal point because the used approach was inapplicable with enough of accuracy in wider area.

The results of the CFD analysis are displayed on the graph in Figure 8. The green curves represent impeller $\mathrm{B}_{1}$ and the orange curves represent impeller $B_{2}$. The chart shows the impact of the impeller blade geometry modification on the hydraulic parameters. Specific energy and efficiency values differed significantly, however, the optimal point of the pump stay unchanged. Pump $B_{1}$ with impeller $\mathrm{B}_{1}$ was expected to produce lower specific energy than pump $B_{2}$ because of the lower blade outlet angle. Nevertheless, the results indicate the opposite. It can be seen that the blade distribution on the back-shroud has, in this case, a stronger impact on the hydraulic parameters than the blade outlet angle.

Experimental measurements of the tested impellers were carried out on a test rig with a close circuit filled with water. Figure 7 shows the whole tested pump. The body of the pump was manufactured by welding of steel parts. Because of the impeller type (semi-open) between the stationary front hub and the rotor (impeller) occurred an axial gap. In order to keep high volume efficiency, which affected the hydraulic efficiency, the gap was reduced to lowest possible distance, however, it was not measured. 
Table 5. Summary of results - values in BEP.

\begin{tabular}{|c|c|c|c|c|c|c|}
\hline & \multicolumn{2}{|c|}{ Impellers $\mathrm{B}_{1-2}$} & \multicolumn{2}{|c|}{ Impeller $B_{3}$} & \multicolumn{2}{|c|}{ Impeller $\mathrm{B}_{4}$} \\
\hline & $\mathrm{B}_{1}$ & $\mathrm{~B}_{2}$ & Meas & Calc & Meas & Calc \\
\hline$Q_{\mathrm{opt}}\left[\mathrm{m}^{3} / \mathrm{s}\right]$ & 0.0105 & 0.0105 & 0.010 & 0.0105 & 0.0095 & 0.0105 \\
\hline$Y_{\text {opt }}[\mathrm{J} / \mathrm{kg}]$ & 98.5 & 80.2 & 128.6 & 96.5 & 130.3 & 95.8 \\
\hline$\eta_{\text {opt }}[\%]$ & 62.4 & 65.4 & 58.9 & 64.2 & 59.5 & 61.7 \\
\hline Vibrations & - & - & Yes & - & No & - \\
\hline
\end{tabular}

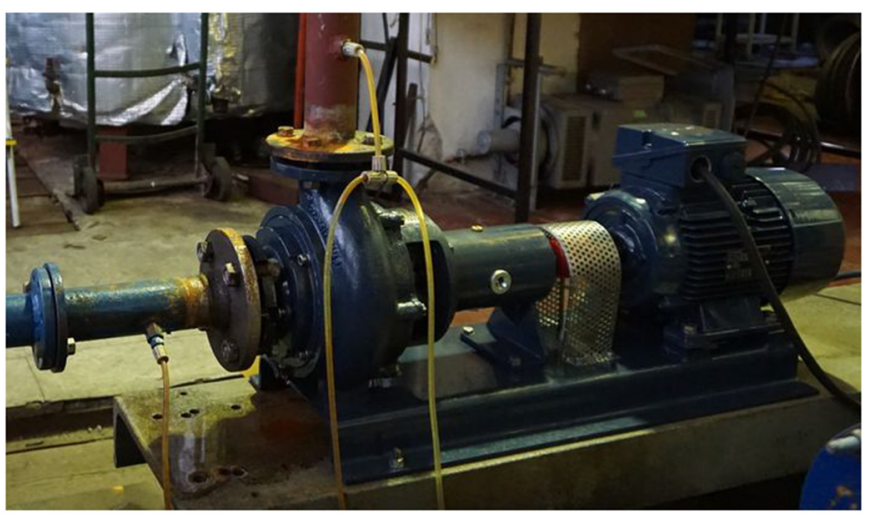

Fig. 7. Investigated pump at the test rig.

The axial gap used in the CFD pump model should cover the real conditions of the flow and in this case, it was estimated at $0.3 \mathrm{~mm}$.

The numerical analysis results and the laboratory measurement results are displayed in Figure 8 as the $Q-Y$ and $Q-\eta$ characteristic curves. The blue curves show laboratory experiment results and the others response to the numerical simulations. In the case of the impellers $\mathrm{B}_{3}$ and $\mathrm{B}_{4}$, it can be seen that the geometry changes (adding and removing of the balancing material) did not influence the location of the best efficiency point and also the hydraulic parameters there. Measured differences between $Q_{\mathrm{opt}}, Y_{\mathrm{opt}}$ and $\eta_{\mathrm{opt}}$ may have its roots in the measurement uncertainty. Even if the measured values show substantial match in the BEP point the $\mathrm{B}_{3}$ and $\mathrm{B}_{4}$ characteristic curve inclinations are different. This causes a substantial increase of $\mathrm{B}_{4}$ impeller specific energy at lower flow rates. The study [3] shows axial and radial force behaviour, which acts on the impellers during the laboratory tests, and when the pump does not operate in the BEP area there is a marked growth of forces. In order to save the test facility and the impeller $\mathrm{B}_{4}$, which is made of the ABS plastic, the measurements are not executed at lower flow rates than $6 \mathrm{~L} / \mathrm{s}$. In the case of the $\mathrm{B}_{4}$ impeller, a higher vibration load can cause a mechanical destruction.

The characteristic curves gained from previous CFD simulations (red colour) indicate certain match with $\mathrm{B}_{3}$ and $\mathrm{B}_{4}$ impeller hydraulic parameters. The results prove a negligible effect of a balancing process on the hydraulic parameters. As it is obvious from Figure 8 the used

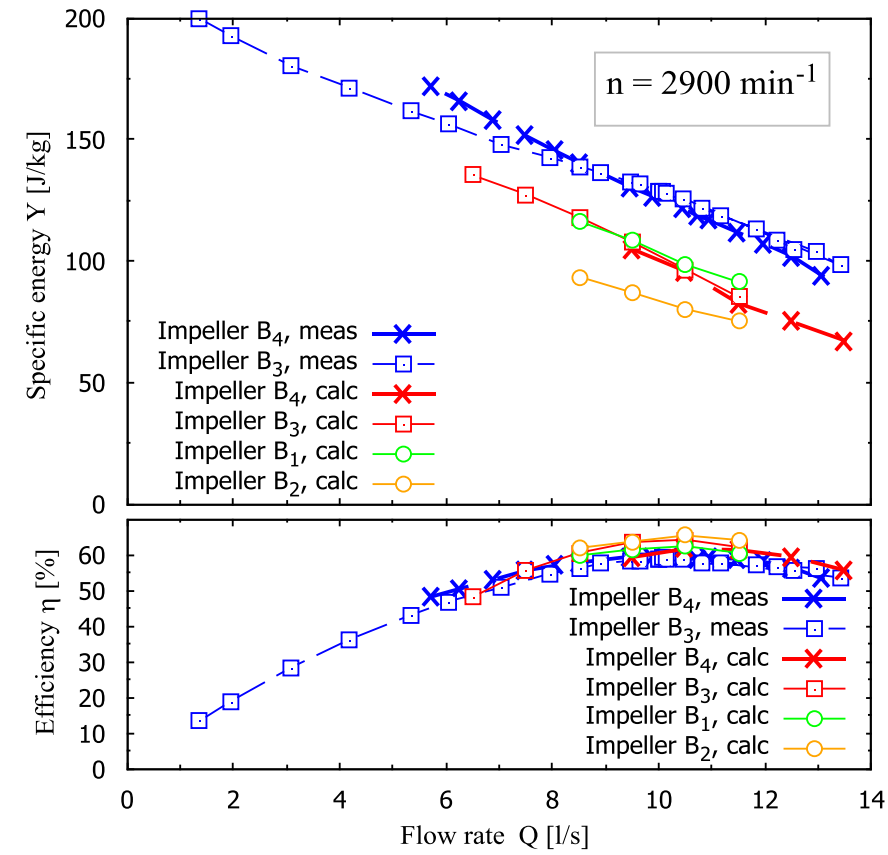

Fig. 8. Results of laboratory measurements and numerical simulations.

interface called Frozen Rotor, which seems to be unsuitable for single-blade pumps, causes a marked difference between the measured and simulated $Q-Y$ curves. This inaccuracy can be solved by applying another frame change option, for example, stage, or by computing in the transient mode.

An overview of attained results is in Table 5. During laboratory tests, some operational properties of the impellers $B_{3}$ and $B_{4}$ have been observed. While the unbalanced impeller $B_{3}$ caused substantial vibrations of the test rig accompanied by a noise the dynamic balanced impeller $\mathrm{B}_{4}$ worked silently without excessive vibrations.

\section{Conclusions}

This paper addresses the impact of impeller geometry changes on the hydraulic parameters of a screw-shaped blade pump and describes the way of designing the singleblade impeller pump so that mechanical and hydraulic property requests, such as efficiency, BEP location, solid 
passage diameter, and dynamic balancing, were fulfilled. A CFD analysis was carried out to find out the hydraulic parameter differences between four pumps with different impeller geometry. The blade outlet angle and the impeller blade arrangement on the back shroud were varied and resulting hydraulic parameter changes were observed. The results of the presented research show the following outcomes:

- The proposed impeller geometry modification, achieved by the change of blade mass distribution, is an effective way to optimize the impeller design for requested hydraulic parameters.

- The impeller blade arrangement on the back shroud has a significant influence on the centre of gravity location, which might be helpful during a subsequent impeller balancing.

- Numerical simulations revealed that the impeller blade displacement modification on the back shroud influenced the hydraulic parameters more significantly than the blade outlet angle change.

- The shape of the blade surface can be designed using basic hydraulic proposal methods but the dynamic balancing of the impeller cannot be ensured. This issue has to be done additionally by adding the balancing mass with the help of CAD software. The effect of balancing masses on the flow has to be monitored by CFD flow simulations.

- The balancing mass can be added so that the total dynamic balancing is achieved and hydraulic parameters remain unchanged, in case of single-blade pumps.

- High passage diameter can be achieved unless the balancing mass is added in a position where the flow channel wide enough is.

- CFD methods of flow simulations are giving a relatively good prediction of hydraulic parameters during the balancing process of the impeller.

\section{Nomenclature}

$A_{2} \quad$ Outlet area $\left[\mathrm{m}^{2}\right]$

$D_{1} \quad$ Inlet diameter [mm]

$D_{2} \quad$ Outlet diameter [mm]

$n \quad$ Speed $\left[\mathrm{min}^{-1}\right]$

$n_{b} \quad$ Specific speed $[-]$

$Q \quad$ Flow rate $\left[1 . \mathrm{s}^{-1}\right]$

$Q_{\text {opt }} \quad$ Flow rate in BEP location $\left[1 . \mathrm{s}^{-1}\right]$

$U_{2} \quad$ Tangential outlet velocity $\left[\mathrm{m} . \mathrm{s}^{-1}\right]$

$x \quad$ Coordinate $[\mathrm{mm}]$

$Y \quad$ Specific energy $\left[\mathrm{J}^{\mathrm{kg}} \mathrm{kg}^{-1}\right]$

\author{
$Y_{\text {opt }} \quad$ Specific energy in BEP location $\left[{\left.\mathrm{J} . \mathrm{kg}^{-1}\right]}^{-1}\right.$ \\ $y \quad$ Coordinate [mm] \\ $\beta_{2} \quad$ Blade outlet angle ['] \\ $\eta \quad$ Efficiency [\%] \\ $\eta_{V} \quad$ Volume efficiency $[\%]$ \\ $\eta_{\text {opt }} \quad$ Maximal efficiency [\%] \\ $\Theta\left(\Phi_{2}\right)$ Hydraulic losses function [-] \\ $\sigma_{y} \quad$ Slip factor [-] \\ $\Phi_{2} \quad$ Dimensionless flow rate [-] \\ $\vartheta \quad$ Cut line inclination $\left[^{\circ}\right]$ \\ $\varphi_{2} \quad$ Wrap angle $\left[{ }^{\circ}\right]$ \\ $\Psi \quad$ Dimensionless specific energy [-] \\ $\Psi_{t} \quad$ Dimensionless theoretical specific energy [-]
}

Acknowledgements. This contribution was created on the basis of the project "Research centre ALLEGRO" (ITMS project code: 26220220198), supported by Operational Programme Research and Development funded by the European Regional Development Fund. The authors gratefully acknowledge the contribution of the Scientific Grant Agency of the Slovak Republic under the grant VEGA $1 / 0743 / 18$.

\section{References}

[1] B. Knížat, Z. Csuka, M. Hyriak, Impeller design of a single blade hydrodynamic pump, AIP Conf. Proc. 1768, 020034 (2016)

[2] M. Kurilla, B. Knížat, Z. Csuka, M. Hyriak, Effect of a blade shape on hydraulic and mechanical properties of a single-blade impeller, MATEC Web Conf. 168, 03001 (2018)

[3] Y. Nishi, J. Fukutomi, R. Fujiwara, Effect of blade outlet angle on radial thrust of single-blade centrifugal pump, IOP Conf. Series: Earth Environ. Sci. 15, 072039 (2012)

[4] X. Cheng, R. Li, Parameter equation study for screw centrifugal pump, Proc. Eng. 31, 914-921 (2012)

[5] J.-H. Kim, B.-M. Cho, Y.-S. Kim, Y.-S. Choi, K.-Y. Kim, J.-H. Kim, Y. Cho, Optimization of a single-channel pump impeller for wastewater treatment, Int. J. Fluid Mach. Syst. 9, 4 (2016)

[6] Y.-L. Zhang, Z.-C. Zhu, H.-S. Dou, B.-L. Cui, Y. L. J.-J. Xiao, A method to determine the slip factor of centrifugal pumps through experiment, Int. J. Turbo Jet-Eng. 32, 59-64 (2014)

[7] Y. Nishi, R. Fujiwara, J. Fukutomi, Design method for singleblade centrifugal pump impeller, J. Fluid Sci. Technol. 4, 3 (2009)

[8] STN EN ISO 9906: Rotodynamic pumps. Hydraulic performance acceptance tests. Grades 1, 2 and 3 (ISO 9906:2012)

Cite this article as: M. Kurilla, B. Knížat, R. Olšiak, P. Slovák, M. Hyriak, Balancing and blade-shaping of a single-blade impeller, Mechanics \& Industry 20, 702 (2019) 